\title{
Intentions and Attempts to Quit JUUL E-Cigarette Use: The Role of Perceived Harm and Addiction
}

\author{
Andréa L. Hobkirk, $\mathrm{PhD}^{1,2,3}$; Brianna Hoglen, $\mathrm{MPH}^{1,3}$; Tianhong Sheng, $\mathrm{BS}^{4}$; Ava Kristich ${ }^{1}$; \\ Jessica M. Yingst, DrPH ${ }^{2,3}$; Kenneth R. Houser, MS ${ }^{1,3}$; Nicolle M. Krebs, MS ${ }^{2,3}$; Sophia I. Allen, PhD ${ }^{2,3}$; \\ Candace R. Bordner, $\mathrm{MS}^{2,3}$; Craig Livelsberger, $\mathrm{BS}^{2,3}$; Jonathan Foulds, $\mathrm{PhD}^{1,3}$
}

\begin{abstract}
Accessible Version: www.cdc.gov/pcd/issues/2022/21_0255.htm
Suggested citation for this article: Hobkirk AL, Hoglen B, Sheng T, Kristich A, Yingst JM, Houser KR, et al. Intentions and Attempts to Quit JUUL E-Cigarette Use: The Role of Perceived Harm and Addiction. Prev Chronic Dis 2022;19:210255. DOI: https://doi.org/10.5888/pcd19.210255.
\end{abstract}

\section{PEER REVIEWED}

\section{Summary}

What is already known on this topic?

Interest in e-cigarette use cessation is growing among regular users, but data on adult e-cigarette users are limited. Qualitative research among young e-cigarette users shows that perceived harm and addictiveness are linked to quit intentions.

What is added by this report?

The current study provides the first quantitative assessment of e-cigarette quit intentions and attempts, and factors associated with quit variables, including perceived e-cigarette harm and addictiveness, among adult users.

What are the implications for public health practice?

Although levels of quit intentions and attempts are low among adult ecigarette users, those with self-reported symptoms of dependence and perceptions that e-cigarettes are more addictive than smoking may benefit from e-cigarette cessation treatments.

\section{Abstract}

\section{Introduction}

Research on electronic cigarette (e-cigarette) quit intentions and attempts is limited despite the potential health benefits of quitting, especially for long-term users. The current study aimed to investigate perceptions of harm and addictiveness and tobacco use characteristics associated with quit variables among users of a popular e-cigarette brand, JUUL.

\section{Methods}

We surveyed 301 US adult JUUL users on their tobacco use characteristics, perceptions of JUUL harm and addictiveness, and quit variables at 3 time points, from July 2019 to April 2020. We used logistic regression models to assess demographic characteristics, smoking characteristics, and perceptions of JUUL harm and addictiveness as correlates of e-cigarette quit intentions, attempts, importance, and confidence.

\section{Results}

Twenty-three percent of the sample had intentions to quit using JUUL within the year, and $22.6 \%$ reported making a lifetime quit attempt. The average rating of quit importance was 4.1 and quit confidence was 5.8 on a Likert scale of 1 to 10 . More than $90 \%$ of the sample indicated that JUUL was at least moderately addictive, whereas less than one-quarter indicated that JUUL was as harmful or more harmful than smoking. Higher levels of perceived JUUL addictiveness were associated with more quit intentions, attempts, and importance. Higher levels of perceived JUUL harm compared with smoking were associated with more quit importance.

\section{Conclusion}

Our findings suggest that a small proportion of adult JUUL users are interested in quitting. Self-reported perceptions of JUUL's addiction potential may be related to more quit attempts. Findings highlight the need for evidence-based information on e-cigarette addictiveness and effective strategies for cessation.

\section{Introduction}

An estimated 8.1 million adults currently use electronic cigarettes (e-cigarettes) in the US (1). Unlike cigarettes, which use combustion to generate tobacco smoke, e-cigarettes aerosolize a nicotinecontaining e-liquid for inhalation (2), resulting in lower intake of carcinogens and toxins for long-term e-cigarette users compared with smokers $(3,4)$. Although replacing cigarettes with e-cigarettes likely reduces health risks in the short term for current smokers, e- 
liquids can contain high levels of nicotine and toxic compounds that may contribute to addiction and respiratory damage $(5,6)$.

Adults' perceptions that e-cigarettes are harmful and addictive have increased over the past decade along with increased messaging about harms (7-9). For example, the Centers for Disease Control and Prevention website states that e-cigarettes contain toxic and carcinogenic chemicals, cause long-lasting changes in the brain, and are not a method for smoking cessation approved by the US Food and Drug Administration (FDA) (eg, cdc.gov/ecigarettes; drugabuse.gov/tobacconicotine-vaping). Data from several national surveys collected in the past decade revealed that the percentage of US adults who believe e-cigarettes are less harmful than cigarettes decreased, while the percentage who believe that ecigarettes are addictive doubled (7-10).

Motivation for e-cigarette use cessation is rising in parallel with attention to the potential harms of e-cigarettes and the growing numbers of long-term e-cigarette users. Online and national surveys of adult e-cigarette users found that approximately $30 \%$ to $60 \%$ expressed some interest in quitting and $10 \%$ to $64 \%$ had made a previous e-cigarette quit attempt (11-13). However, research is limited on the factors associated with e-cigarette quit intentions and attempts, including the influence of harm or addiction perceptions.

Given the changing landscape of e-cigarette harm messaging and regulations, and the media attention given to e-cigarettes, the primary objective of the current study was to investigate ecigarette quit intentions and attempts by identifying associated factors and focusing on adult JUUL users. Of all e-cigarette brands, JUUL has received the most negative attention because of its popularity among adolescents and young adults and its relatively high levels of nicotine (14). Therefore, we hypothesized that perceptions of JUUL's addictive potential and harm relative to cigarettes would be associated with quit intentions and attempts.

\section{Methods}

In this cross-sectional survey, we used Amazon Mechanical Turk (MTurk) to recruit adults aged 18 or older who indicated having used JUUL in the past 30 days. MTurk is an online labor market where individuals with a registered account, called workers, can complete online jobs, such as computer tasks and surveys, for compensation. Workers can choose from a list of jobs available to them based on their demographic characteristics and geographic location. Substance users and young adults tend to be overrepresented among MTurk workers compared with the general population, making MTurk an ideal platform for research on e-cigarette use behavior (15). To quantify workers' performance, MTurk assigns each worker a job approval rating based on their number of successfully completed jobs. To improve the reliability and validity of the current survey, we implemented a requirement that only US workers with job approval ratings of at least $98 \%$ were eligible to participate. We conducted the cross-sectional survey at 3 time points, July 2019, January 2020, and April 2020, with different samples at each point. All participants provided informed consent electronically before completing study procedures and were compensated \$2 for participation. This payment is consistent with MTurk standards (16). All procedures were approved by the Penn State College of Medicine Institutional Review Board.

\section{Measures}

The 30-minute survey was developed by the study team to assess JUUL use behavior. The survey assessed demographic information with the items, "How old are you?" and "Are you male or female?"; JUUL use characteristics, including "How long have you been using an electronic cigarette?" and "How long have you been using a JUUL electronic cigarette?" with options to respond in the number of days, months, or years; "How many days in the last 30 have you used your JUUL electronic cigarette?"; "In the past 30 days, what nicotine concentration JUUL pods did you purchase/ use?" with response options " $5 \%$," "3\%," or "both"; "Where did you purchase your JUUL electronic cigarette?" with response options "gas station," "vape shop," "tobacco store," "online," or "I did not purchase"; and "When was the last time you smoked a cigarette?" with options to respond in the number or days, months, or years. Former smokers were asked, "Did you quit smoking before or after you started using JUUL?" with response options "I quit cigarette smoking before I started using JUUL or any other electronic cigarette," "I quit cigarette smoking after I started using an e-cig other than JUUL," and "I quit cigarette smoking after I started using JUUL." We measured e-cigarette dependence with the Penn State Electronic Cigarette Dependence Index (PSECDI); total scores for this index range from 0 to 20 , with higher scores indicating greater dependence. Levels of dependence were categorized according to the PSECDI as not dependent (0-3), low dependence (4-8), medium dependence (9-12), and high dependence $(\geq 13)$ (17). The PSECDI has normative data from more than 3,600 e-cigarette users and shows construct validity in that scores are related to the nicotine concentration of liquids used and show convergent validity with the E-cigarette dependence scale among exclusive e-cigarette users $(r=0.71)(18)$. We used the following PSECDI item as an independent correlate in our analyses: "How many times per day do you use your JUUL electronic cigarette? (assume that one time consists of around 15 puffs or lasts around 10 minutes)."

Items assessing the quit variables included 1) "Are you planning to continue using your JUUL electronic cigarette for at least the next year, or quit within that time frame?" with response options

The opinions expressed by authors contributing to this journal do not necessarily reflect the opinions of the U.S. Department of Health and Human Services, the Public Health Service, the Centers for Disease Control and Prevention, or the authors' affiliated institutions. 
"I'll quit using JUUL within a year," "I plan to continue using the JUUL," or "Don't know"; 2) "How important is it for you to quit electronic cigarette use now?" on a 10-point Likert scale ranging from "not at all" to "very"; 3) "How confident are you that you could quit electronic cigarette use now?" on a 10-point Likert scale ranging from 1 ("not at all") to 10 ("very"); and 4) "Have you ever tried to quit using your JUUL electronic cigarette?" with response options no or yes. Those who made a quit attempt were asked the duration of their quit attempt and if they used any of the following methods during the attempt: "nicotine replacement therapy," "cold turkey," "used another tobacco product," or "FDAapproved medication like Zyban or Chantix." Participants responded to 2 questions about their perceptions of JUUL use: 1) "Overall, how addictive do you believe using a JUUL is?" with response options "not at all addictive," "moderately addictive," and "very addictive" and 2) "Compared to smoking, would you say that JUUL use is ..." with response options "much less harmful," "less harmful," "just as harmful," or "much more harmful."

The survey also inquired about education and employment, eliquid and pod purchases and refilling behaviors, physical and health effects of JUUL use, motivations for JUUL use, and use of other tobacco products. Information from respondents on the use of flavored JUUL pods in the context of flavor regulations is available elsewhere (19).

\section{Data cleaning}

To exclude respondents who were not authentic JUUL users, we required all participants to submit a nonstock photograph of their JUUL device. We excluded from analysis respondents who submitted a stock or non-JUUL photo and illogical or random responses $(\mathrm{n}=98)$. In addition, we removed from analysis $22 \mathrm{du}-$ plicate responses across the 3 time points. In total, we excluded 120 respondents from analysis, which resulted in a final sample of 301 unique respondents.

\section{Data analysis}

We used SPSS version 26 (IBM Corp) to calculate means and frequencies and to conduct 1-way analyses of variance and $\chi^{2}$ tests for all demographic, perception, and quit variables by smoking status. To prepare the data for regression analysis, we normalized positively skewed variables by applying a square root transformation to the number of months of e-cigarette and JUUL use and a natural log transformation to the number of JUUL use times per day. For smoking status, current smokers were those that indicated using a cigarette less than 1 month ago. Former smokers indicated last using a cigarette 1 month ago or longer. Never smokers reported never using a cigarette. We categorized scores on self-reported quit importance and confidence into low (1-3), medium (4-7), and high (8-10) to simplify analysis. We conducted binomial and multinomial logistic regression models to examine 1) demographic and smoking characteristics as correlates of harm and addiction perceptions and 2) demographic and smoking characteristics and harm and addiction perceptions as correlates of quit variables (ie, intentions, attempts, importance, and confidence). The correlates assessed were sex, age, survey collection time point, place of device purchase, smoking status (ie, current, former, never), PSECDI level, number of use times per day, days of use in the past 30 , months of e-cigarette use, months of JUUL use, perceived harm, and perceived addictiveness. We treated all correlates as fixed effects in the models. We first fit each model by using all correlates and then selected models based on the Akaike information criterion (AIC). The AIC considers the risk of overfitting and underfitting to converge on a final model with good fit for the data and simplicity. We calculated total fit metrics (AIC and residual deviance) for each model. We calculated analysis of deviance tables to display the significance of each correlate in the final models with $\chi^{2}$ tests. We calculated odds ratios and significance values of each model parameter estimate to assist with interpretation; however, some subgroup sample sizes were too small to produce reliable parameter estimates, and therefore should be interpreted with caution. We conducted regression analyses by using R statistical software version 3.4.2 (R Foundation for Statistical Computing).

\section{Results}

The final sample included 301 current JUUL users; $36.2 \%$ (n = 109) were women, and the mean age was 31.9 (SD, 8.5) (Table 1). The sample included 55 (18.3\%) current smokers, $165(54.8 \%)$ former smokers, and $81(26.9 \%)$ never smokers. Former smokers last smoked a cigarette 24.6 (SD, 30.7) months ago on average; $35 \%$ (58 of 165) of former smokers reported that they quit using cigarettes after starting to use a JUUL. Half of the sample (51\%, n $=154$ ) reported using their JUUL daily with an average of 11.8 (SD, 37.9) times per day. Self-reported JUUL use ranged from 1 to 500 times per day among the total sample. Four participants reported using their JUUL 100 or more times per day.

\section{Total sample quit variables and harm perceptions}

Less than one-quarter $(\mathrm{n}=68 ; 22.6 \%)$ indicated an intention to quit using JUUL within the year. The sample rated a mean of 4.1 (SD, 2.8) of 10 for the importance of quitting and a mean of 5.8 (SD, 2.7) of 10 for confidence in their ability to quit. Of the 68 $(22.6 \%)$ who made a lifetime JUUL quit attempt, the average attempt lasted 2.4 months (SD, 3.4) and almost half $(\mathrm{n}=31 ; 45.6 \%)$

The opinions expressed by authors contributing to this journal do not necessarily reflect the opinions of the U.S. Department of Health and Human Services, the Public Health Service, the Centers for Disease Control and Prevention, or the authors' affiliated institutions. 
reported an attempt that lasted less than a month. Of those who reported a quit attempt, most $(n=46 ; 67.6 \%)$ reported quitting JUUL "cold turkey" with the remainder reporting use of nicotine replacement therapy $(\mathrm{n}=11 ; 16.2 \%)$, another tobacco product $(\mathrm{n}=$ $7 ; 10.3 \%)$, or an FDA-approved medication $(\mathrm{n}=4 ; 5.9 \%)$.

Two-thirds of the sample indicated that JUUL was moderately addictive $(\mathrm{n}=202 ; 67.1 \%)$ and one-quarter indicated that JUUL was very addictive $(n=75 ; 24.9 \%) ; 76.1 \%(n=229)$ of the sample believed that JUUL was less or much less harmful than smoking, and only $10(3.3 \%)$ participants believed that JUUL was much more harmful than smoking (Table 1).

Of the 75 JUUL users who believed use was very addictive, 45 $(60.0 \%)$ indicated they would continue using JUUL (Figure 1). Among JUUL users who believed use was very addictive, the median PSECDI score was 12.0 (range, 2-18), and among users who believed use was not at all addictive, the median PSECDI score was 5 (range, 0-11) (Figure 2). Among JUUL users who believed use was much more harmful than smoking, the median score was 11 (range, 2-18), and among users who believed use was much less harmful than smoking, the median score was 8 (range, $0-18$ ).
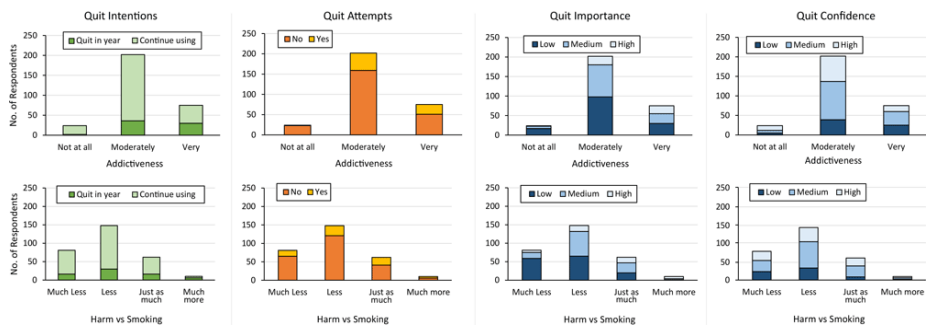

Figure 1. Frequency of quit variables by harm and addiction perceptions among a sample of US adult JUUL users ( $N=301), 2019-2020$.
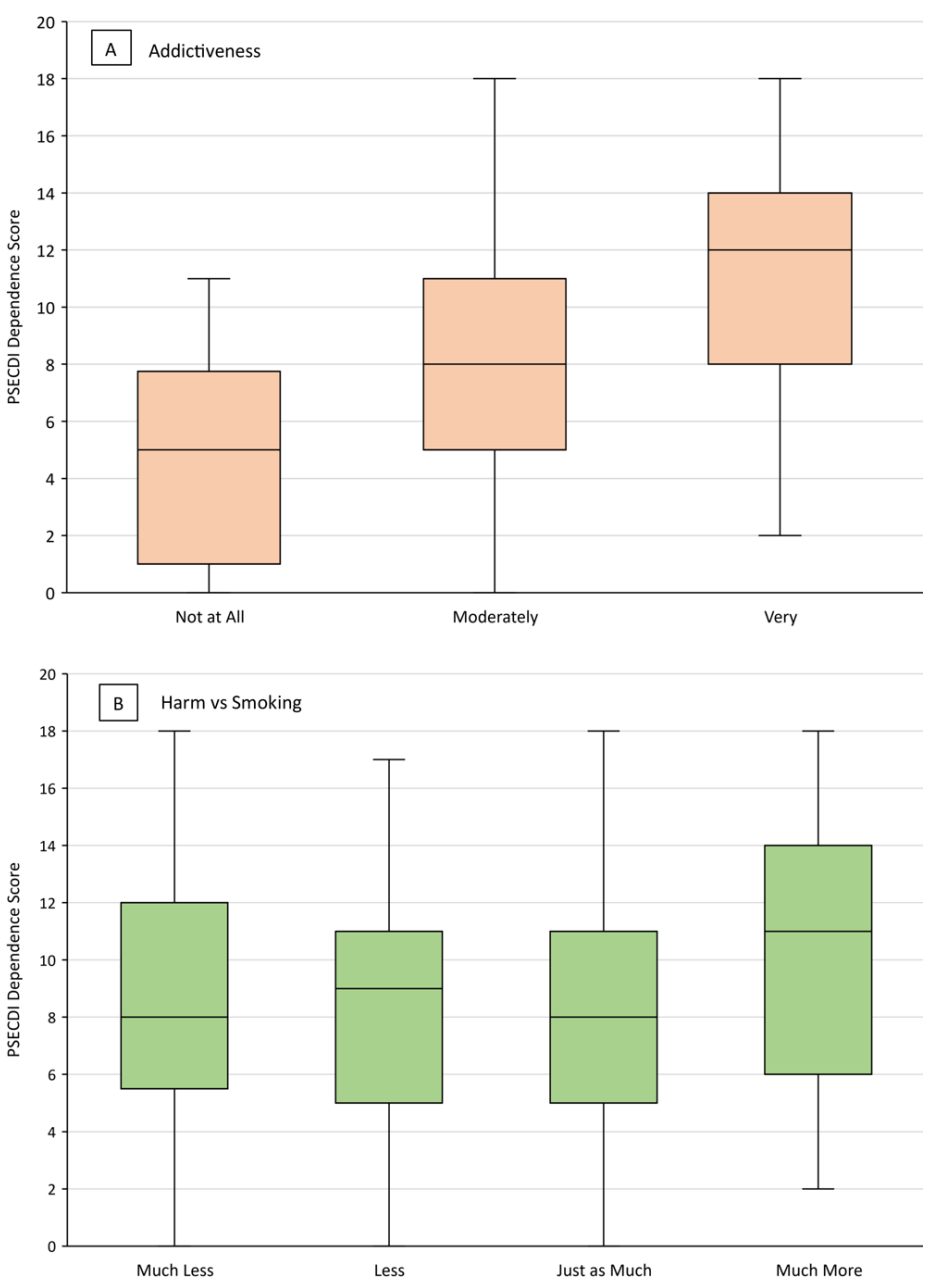

Figure 2. Mean PSECDI dependence score by level of A) perceived addictiveness of JUUL and B) perceived harm (compared with smoking) of JUUL among a sample of US adult JUUL users $(N=301), 2019-2020$. Boxes indicate the 25th percentile, median, and 75th percentile. Whiskers show the minimum and maximum ranges. Abbreviation: PSECDI, Penn State Electronic Cigarette Dependence Index.

\section{Regression models of JUUL perceptions and quit variables}

In the final logistic regression models, factors associated with higher perceived JUUL addictiveness included higher PSECDI score, being female (vs male), and being a current or former smoker (vs never smoker) (Table 2). Older age was associated with a higher likelihood of endorsing JUUL as moderately (vs not at all) addictive but a lower likelihood of endorsing JUUL as very (vs not at all) addictive. Factors associated with higher perceived JUUL harm compared with smoking included fewer lifetime months of e-cigarette use, being a current or former smoker (vs never smoker), and being female (vs male) (Table 3). Factors as-

\footnotetext{
The opinions expressed by authors contributing to this journal do not necessarily reflect the opinions of the U.S. Department of Health and Human Services, the Public Health Service, the Centers for Disease Control and Prevention, or the authors' affiliated institutions.
} 
sociated with intentions to quit JUUL use in the next year included higher perceived addictiveness and fewer times per day of JUUL use (Table 4). Factors associated with a lifetime quit attempt included higher perceived addictiveness, higher PSECDI score, being a never smoker (vs current or former smoker), and fewer days of JUUL use in the past 30 days (Table 5). Factors associated with higher quit importance included higher perceived addictiveness and harm, higher PSECDI score, being in the second or third collection time point (vs first time point), fewer days of JUUL use in the past 30 days, and fewer lifetime months of ecigarette use (Table 6). Place of JUUL purchase was a relevant correlate of perceived quit importance, but the direction was difficult to interpret because of sample sizes for the purchase categories. Lower dependence level was the only significant correlate of higher quit confidence.

\section{Discussion}

The current study surveyed adult JUUL users on their quit intentions, attempts, importance, and confidence and their perceptions of JUUL harm and addictiveness in 3 unique groups at 3 time points. A minority of our participants reported an intention to quit JUUL in the upcoming year or that they had ever attempted to quit JUUL use. Most of our sample perceived e-cigarettes to be less harmful than smoking but at least moderately addictive. Public health warnings about e-cigarette addictiveness have targeted JUUL because of its ability to deliver nicotine levels more similar to a combustible cigarette and at higher levels than most other ecigarettes on the market (20). The actual addiction potential of ecigarettes, however, remains under debate because levels of selfreported dependence on e-cigarettes are consistently lower among e-cigarettes users compared with cigarette smokers $(17,21,22)$. In our sample, perceived addictiveness increased with level of dependence, suggesting that participants' perceptions of JUUL addictiveness may be directly informed by awareness of their own addiction to e-cigarettes.

Higher perceived addictiveness was associated with more quit intentions, attempts, and importance, while higher perceived harm compared with smoking was associated only with more quit importance. Previous qualitative studies of adolescents and young adults found harm and addiction perceptions were primary reasons for future intentions to quit e-cigarette use and previous quit attempts $(23,24)$. The link between addiction perceptions and quit intentions suggests that messaging about the addictiveness of ecigarettes could increase public interest in quitting among adults, even for smokers using them as cigarette alternatives. This highlights the need for more evidence-based information about the ad- dictiveness of e-cigarettes, including which e-cigarette product features or user characteristics increase risk for e-cigarette addiction.

Reported quit importance was highest in the third and final survey time point, which occurred in April 2020, after the e-cigarette or vaping use-associated lung injury (EVALI) outbreak that began in July 2019 and resolution of its cause (ie, black market tetrahydrocannabinol [THC] products contaminated with vitamin E acetate) in February 2020 (25). We did not measure exposure to EVALI information; however, we suspect that the increased media attention to the potential harms of e-cigarettes in late 2019 may have contributed to the increased quit importance in the survey data collected in January 2020. Although we did not find a significant increase in harm perceptions in our later cohorts, our assessment of relative harm compared with smoking may have missed JUUL users' perceptions of general harm. Alternatively, the COVID-19 pandemic led to major shutdowns and a surge of health information in the US in March 2020, just before our final survey in April 2020. The increase in quit importance we observed may have been related to the attention to respiratory health and tobacco use during the pandemic and not e-cigarette messaging.

Higher e-cigarette dependence as measured on PSECDI was associated with more quit attempts, higher quit importance, and lower quit confidence, but not quit intentions. This finding, combined with the positive association between dependence and perceived addictiveness, suggests that JUUL users with higher levels of dependence are aware of their own difficulties with quitting and might be open to e-cigarette cessation interventions, even if they are not planning to quit on their own. Simply inquiring about interest in e-cigarette cessation or administering brief self-report dependence measures in clinical and community settings could be an easy and effective way to identify e-cigarette users who might be willing to make a quit attempt. However, our data show that quitting confidence is not high among JUUL users in general and is even lower among those with high levels of dependence. This points to the need for interventions that can boost quitting confidence and success.

Empirically supported treatments of e-cigarette cessation are lacking. A pilot trial showed promise for nicotine replacement therapy, tapering use, and self-guided methods for e-cigarette cessation among young adults (26). A randomized clinical trial among more than 2,000 young adult e-cigarette users found better cessation rates in a text-messaging program $(24.1 \%)$ than in an assessmentonly control condition after 7 months $(18.6 \%)(27)$. The cessation program, This is Quitting, used a combination of social support and cognitive and behavioral coping skills training conducted entirely through text messages (27). Among our sample, those who

The opinions expressed by authors contributing to this journal do not necessarily reflect the opinions of the U.S. Department of Health and Human Services, the Public Health Service, the Centers for Disease Control and Prevention, or the authors' affiliated institutions. 
attempted to quit primarily did it "cold turkey" and almost half of those who attempted reported that cessation lasted less than a month. Most previous research has focused on links between ecigarette use and smoking cessation, rather than e-cigarette cessation (28). More research is needed to develop effective evidencebased cessation interventions, to increase awareness of e-cigarette cessation options among the public, and to identify those most in need of cessation programs.

The primary limitations of the current study are the small sample size and use of convenience sampling through MTurk. MTurk participants are not representative of the general US population, which limits our ability to make any population-based inferences from our results (29). However, MTurk is an ideal platform to recruit e-cigarette users because workers tend to be young and overrepresent substance users (30). The anonymity of MTurk facilitates honest and unbiased responses when stigmatized behaviors are being assessed, which cannot always be achieved in faceto-face laboratory studies. We also used numerous data validity and reliability checks. As with any survey study, we were not able to biochemically verify that participants were nicotine users. Purposely masking the true eligibility criteria for the study helped to identify respondents who were not current e-cigarette users. Participants had little incentive to lie about their use, given the many work opportunities on MTurk.

In conclusion, we found that e-cigarette quit intentions and attempts were low among our sample of US adult JUUL users and were associated with higher perceptions of JUUL addictiveness. Increased awareness of the potential addictiveness of e-cigarettes may drive quit intentions and attempts; however, more empirical data on e-cigarette addictiveness is needed to accurately inform the public. Public health priorities should include continuing to educate the public on the known risks of e-cigarettes, their potential role in assisting smoking cessation (31), developing methods for identifying and engaging those at risk for e-cigarette dependence, and developing effective and accessible methods for ecigarette cessation.

\section{Acknowledgments}

A.L.H. is supported by a National Institute on Drug Abuse (NIDA) career development award (K23-DA045081). S.I.A. is supported by a NIDA Diversity supplement (U01-DA04551701S2). J.F., J.M.Y., N.M.K., C.R.B., and C.L. are supported by NIDA grants (U01-DA045517 and R01 DA048428). J.F. has received grants from the National Institutes of Health (NIH); has previously received a grant, personal fees, and nonfinancial support (eg, study drug) from Pfizer Inc, unrelated to this article; in the past ( $>3$ years ago), has done paid consulting for pharmaceut- ical companies involved in manufacturing smoking-cessation medications (eg, GlaxoSmithKline, Johnson \& Johnson); and has acted as a deposed and compensated expert witness on behalf of plaintiffs suing cigarette manufacturers. The authors have no other conflicts of interest to disclose. This project was facilitated by the Penn State Center for Research on Tobacco and Health. The content is solely the responsibility of the authors and does not necessarily represent the official views of NIH. No copyrighted material, surveys, instruments, or tools were used in this article.

\section{Author Information}

Corresponding Author: Andréa L. Hobkirk, PhD, Pennsylvania State University College of Medicine, 500 University Dr, Mail Code CH69, Hershey, PA 17033. Telephone: 717-531-0003 ext. 286415. Email: ahobkirk@pennstatehealth.psu.edu.

Author Affiliations: ${ }^{1}$ Department of Psychiatry and Behavioral Health, Pennsylvania State University College of Medicine, Hershey, Pennsylvania. ${ }^{2}$ Department of Public Health Sciences, Pennsylvania State University College of Medicine, Hershey, Pennsylvania. ${ }^{3}$ Center for Research on Tobacco and Health, Pennsylvania State University College of Medicine, Hershey, Pennsylvania. ${ }^{4}$ Department of Statistics, Pennsylvania State University, State College, Pennsylvania.

\section{References}

1. Villarroel MA, Cha AE, Vahratian A. Electronic cigarette use among U.S. adults, 2018. NCHS Data Brief 2020;(365):1-8.

2. Breland A, Soule E, Lopez A, Ramoa C, El-Hellani A, Eissenberg T. Electronic cigarettes: what are they and what do they do? Ann N Y Acad Sci 2017;1394(1):5-30.

3. Shahab L, Goniewicz ML, Blount BC, Brown J, McNeill A, Alwis KU, et al. Nicotine, carcinogen, and toxin exposure in long-term e-cigarette and nicotine replacement therapy users: a cross-sectional study. Ann Intern Med 2017;166(6):390-400.

4. McRobbie H, Phillips A, Goniewicz ML, Smith KM, KnightWest O, Przulj D, et al. Effects of switching to electronic cigarettes with and without concurrent smoking on exposure to nicotine, carbon monoxide, and acrolein. Cancer Prev Res (Phila) 2015;8(9):873-8.

5. Lee MS, Allen JG, Christiani DC. Endotoxin and ( $1 \rightarrow 3)-\beta$-Dglucan contamination in electronic cigarette products sold in the United States. Environ Health Perspect 2019;127(4):47008.

6. Hajek P, Pittaccio K, Pesola F, Myers Smith K, Phillips-Waller A, Przulj D. Nicotine delivery and users' reactions to Juul compared with cigarettes and other e-cigarette products. Addiction 2020;115(6):1141-8.

The opinions expressed by authors contributing to this journal do not necessarily reflect the opinions of the U.S. Department of Health and Human Services, the Public Health Service, the Centers for Disease Control and Prevention, or the authors' affiliated institutions. 
7. Tan AS, Bigman CA. E-cigarette awareness and perceived harmfulness: prevalence and associations with smokingcessation outcomes. Am J Prev Med 2014;47(2):141-9.

8. Majeed BA, Weaver SR, Gregory KR, Whitney CF, Slovic P, Pechacek TF, et al. Changing perceptions of harm of ecigarettes among U.S. adults, 2012-2015. Am J Prev Med 2017;52(3):331-8.

9. Huerta TR, Walker DM, Mullen D, Johnson TJ, Ford EW. Trends in e-cigarette awareness and perceived harmfulness in the U.S. Am J Prev Med 2017;52(3):339-46.

10. Huang J, Feng B, Weaver SR, Pechacek TF, Slovic P, Eriksen MP. Changing perceptions of harm of e-cigarette vs cigarette use among adults in 2 US national surveys from 2012 to 2017. JAMA Netw Open 2019;2(3):e191047.

11. Rosen RL, Steinberg ML. Interest in quitting e-cigarettes among adults in the United States. Nicotine Tob Res 2020; 22(5):857-8.

12. Etter JF. Are long-term vapers interested in vaping cessation support? Addiction 2019;114(8):1473-7.

13. Garey L, Mayorga NA, Peraza N, Smit T, Nizio P, Otto MW, et al. Distinguishing characteristics of e-cigarette users who attempt and fail to quit: dependence, perceptions, and affective vulnerability. J Stud Alcohol Drugs 2019;80(1):134-40.

14. Huang J, Duan Z, Kwok J, Binns S, Vera LE, Kim Y, et al. Vaping versus JUULing: how the extraordinary growth and marketing of JUUL transformed the US retail e-cigarette market. Tob Control 2019;28(2):146-51.

15. Strickland JC, Stoops WW. The use of crowdsourcing in addiction science research: Amazon Mechanical Turk. Exp Clin Psychopharmacol 2019;27(1):1-18.

16. Auer EM, Behrend TS, Collmus AB, Landers RN, Miles AF. Pay for performance, satisfaction and retention in longitudinal crowdsourced research. PLoS One 2021;16(1):e0245460.

17. Foulds J, Veldheer S, Yingst J, Hrabovsky S, Wilson SJ, Nichols TT, et al. Development of a questionnaire for assessing dependence on electronic cigarettes among a large sample of ex-smoking e-cigarette users. Nicotine Tob Res 2015;17(2):186-92.

18. Morean ME, Krishnan-Sarin S, Sussman S, Foulds J, Fishbein $\mathrm{H}$, Grana R, et al. Psychometric evaluation of the e-cigarette dependence scale. Nicotine Tob Res 2019;21(11):1556-64.

19. Yingst JM, Bordner CR, Hobkirk AL, Hoglen B, Allen SI, Krebs NM, et al. Response to flavored cartridge/pod-based product ban among adult JUUL users: "You get nicotine however you can get it." Int J Environ Res Public Health 2020; 18(1):E207.

20. Yingst JM, Hrabovsky S, Hobkirk A, Trushin N, Richie JP Jr, Foulds J. Nicotine absorption profile among regular users of a pod-based electronic nicotine delivery system. JAMA Netw Open 2019;2(11):e1915494.
21. Shiffman S, Sembower MA. Dependence on e-cigarettes and cigarettes in a cross-sectional study of US adults. Addiction 2020;115(10):1924-31.

22. Liu G, Wasserman E, Kong L, Foulds J. A comparison of nicotine dependence among exclusive e-cigarette and cigarette users in the PATH study. Prev Med 2017;104:86-91.

23. Amato MS, Bottcher MM, Cha S, Jacobs MA, Pearson JL, Graham AL. "It's really addictive and I'm trapped:" a qualitative analysis of the reasons for quitting vaping among treatment-seeking young people. Addict Behav 2021; 112:106599.

24. Hanafin J, Clancy L. A qualitative study of e-cigarette use among young people in Ireland: incentives, disincentives, and putative cessation. PLoS One 2020;15(12):e0244203.

25. Blount BC, Karwowski MP, Shields PG, Morel-Espinosa M, Valentin-Blasini L, Gardner M, et al. Vitamin E acetate in bronchoalveolar-lavage fluid associated with EVALI. N Engl J Med 2020;382(8):697-705.

26. Sahr M, Kelsh S, Blower N, Sohn M. Pilot study of electronic nicotine delivery systems (ENDS) cessation methods. Pharmacy (Basel) 2021;9(1):21.

27. Graham AL, Amato MS, Cha S, Jacobs MA, Bottcher MM, Papandonatos GD. Effectiveness of a vaping cessation text message program among young adult e-cigarette users: a randomized clinical trial. JAMA Intern Med 2021; 181(7):923-30.

28. Berry KM, Reynolds LM, Collins JM, Siegel MB, Fetterman JL, Hamburg NM, et al. E-cigarette initiation and associated changes in smoking cessation and reduction: the Population Assessment of Tobacco and Health Study, 2013-2015. Tob Control 2019;28(1):42-9.

29. Huff C, Tingley D. "Who are these people?" Evaluating the demographic characteristics and political preferences of MTurk survey respondents. Res Politics 2015;2015(3):1-12.

30. Shapiro DN, Chandler J, Mueller PA. Using Mechanical Turk to study clinical populations. Clin Psychol Sci 2013; 1(2):213-20.

31. Hajek P, Phillips-Waller A, Przulj D, Pesola F, Myers Smith $\mathrm{K}$, Bisal $\mathrm{N}$, et al. A randomized trial of e-cigarettes versus nicotine-replacement therapy. N Engl J Med 2019; 380(7):629-37.

The opinions expressed by authors contributing to this journal do not necessarily reflect the opinions of the U.S. Department of Health and Human Services, the Public Health Service, the Centers for Disease Control and Prevention, or the authors' affiliated institutions. 


\section{Tables}

Table 1. Demographic Characteristics, JUUL Use, JUUL Perceptions, and Quit Variables Across Smoking Status for a Sample of US Adult JUUL Users, 2019-2020 ${ }^{a}$

\begin{tabular}{|c|c|c|c|c|c|}
\hline Characteristic & $\begin{array}{l}\text { Never smoker } \\
(n=81)\end{array}$ & $\begin{array}{l}\text { Current smoker } \\
(n=55)\end{array}$ & $\begin{array}{l}\text { Former smoker } \\
(n=165)\end{array}$ & $\begin{array}{l}\text { Total sample } \\
(\mathrm{N}=301)\end{array}$ & $P$ value ${ }^{b}$ \\
\hline \multicolumn{6}{|l|}{ Demographic } \\
\hline Female sex, n (\%) & $35(43.2)$ & $13(23.6)$ & $61(37.0)$ & $109(36.2)$ & .06 \\
\hline Age, mean (SD), y & $28.8(8.0)$ & $35.2(9.5)$ & $32.3(7.9)$ & $31.9(8.5)$ & $<.001$ \\
\hline \multicolumn{6}{|l|}{ JUUL use } \\
\hline $\begin{array}{l}\text { No. of months have used e-cigarettes, mean } \\
\text { (SD) }\end{array}$ & $20.9(21.8)$ & $28.7(22.4)$ & $29.8(24.6)$ & $27.2(23.7)$ & .02 \\
\hline No. of months have used JUUL, mean (SD) & $13.5(13.0)$ & $13.4(12.5)$ & $14.0(10.6)$ & $13.8(11.6)$ & .93 \\
\hline JUUL use days in past 30 days, mean (SD) & $21.1(8.6)$ & $22.8(8.7)$ & $24.5(8.3)$ & $23.3(8.6)$ & .01 \\
\hline JUUL use times per day, mean (SD) & $10.3(17.4)$ & $16.2(53.3)$ & $11.2(39.1)$ & $11.8(37.9)$ & .63 \\
\hline \multicolumn{6}{|l|}{ Where purchase JUUL, n (\%) } \\
\hline Gas station & $22(27.2)$ & $22(40.0)$ & $63(38.2)$ & $107(35.5)$ & \multirow[t]{5}{*}{.06} \\
\hline Vape shop & $28(34.6)$ & $9(16.4)$ & $52(31.5)$ & 89 (29.6) & \\
\hline Tobacco store & $7(8.6)$ & $9(16.4)$ & $14(8.5)$ & $30(10.0)$ & \\
\hline Online & $23(28.4)$ & $11(20.0)$ & $30(18.2)$ & $64(21.3)$ & \\
\hline Did not purchase & $1(1.2)$ & $4(7.3)$ & $6(3.6)$ & $11(3.7)$ & \\
\hline \multicolumn{6}{|l|}{ Nicotine concentration, n (\%) } \\
\hline $5 \%$ concentration & $32(42.7)$ & $33(63.5)$ & $81(51.9)$ & $146(51.6)$ & \multirow[t]{3}{*}{.07} \\
\hline $3 \%$ concentration & 31 (41.3) & $11(21.2)$ & $42(26.9)$ & $84(29.7)$ & \\
\hline Both & $12(16.0)$ & $8(15.4)$ & $33(21.2)$ & $53(18.7)$ & \\
\hline PSECDI total score, mean $(S D)^{C}$ & $8.0(4.5)$ & $9.1(4.4)$ & $8.4(4.0)$ & $8.4(4.2)$ & .31 \\
\hline \multicolumn{6}{|l|}{ PSECDI dependence level, n (\%) } \\
\hline Not dependent & $17(21.0)$ & $8(14.5)$ & $20(12.1)$ & $45(15.0)$ & \multirow[t]{4}{*}{.43} \\
\hline Low dependence & 27 (33.3) & $15(27.3)$ & $63(38.2)$ & 105 (34.9) & \\
\hline Medium dependence & $25(30.9)$ & $19(34.5)$ & $53(32.1)$ & $97(32.2)$ & \\
\hline High dependence & $12(14.8)$ & $13(23.6)$ & $29(17.6)$ & $54(17.9)$ & \\
\hline \multicolumn{6}{|l|}{ JUUL perceptions } \\
\hline \multicolumn{6}{|l|}{ How addictive, $n(\%)$} \\
\hline Not at all addictive & $14(17.3)$ & $4(7.3)$ & $6(3.6)$ & $24(8.0)$ & \multirow[t]{3}{*}{.002} \\
\hline Moderately addictive & $54(66.7)$ & $37(67.3)$ & $111(67.3)$ & 202 (67.1) & \\
\hline Very addictive & $13(16.0)$ & $14(25.5)$ & 48 (29.1) & 75 (24.9) & \\
\hline
\end{tabular}

Abbreviation: PSECDI, Penn State Electronic Cigarette Dependence Index.

${ }^{a}$ Data source: A 30-minute survey was developed by the study team and administered at 3 time points: July 2019, January 2020 , and April 2020.

${ }^{\mathrm{b}} P$ values determined from variable comparisons across smoking status using 1-way analyses of variance and $x^{2}$ tests.

${ }^{\mathrm{c}}$ Scores range from 0 to 20 , where higher scores indicate greater dependence. Levels of dependence were categorized as not dependent (0-3), low dependence (4-8), medium dependence (9-12), and high dependence ( $\geq 13)(17)$.

d A 10-point Likert scale ranging from "not at all" to "very." Scores categorized into low (1-3), medium (4-7), and high (8-10) to simplify analysis. 
(continued)

Table 1. Demographic Characteristics, JUUL Use, JUUL Perceptions, and Quit Variables Across Smoking Status for a Sample of US Adult JUUL Users, 2019-2020 ${ }^{a}$

\begin{tabular}{|c|c|c|c|c|c|}
\hline Characteristic & $\begin{array}{l}\text { Never smoker } \\
(n=81)\end{array}$ & $\begin{array}{l}\text { Current smoker } \\
(n=55)\end{array}$ & $\begin{array}{l}\text { Former smoker } \\
(n=165)\end{array}$ & $\begin{array}{l}\text { Total sample } \\
(\mathrm{N}=301)\end{array}$ & $P$ value ${ }^{b}$ \\
\hline Much less harmful & $30(37.0)$ & $8(14.5)$ & $43(26.1)$ & $81(26.9)$ & \multirow[t]{4}{*}{.04} \\
\hline Less harmful & $29(35.8)$ & $31(56.4)$ & $88(53.3)$ & $148(49.2)$ & \\
\hline Just as harmful & $18(22.2)$ & $13(23.6)$ & $31(18.8)$ & $62(20.6)$ & \\
\hline Much more harmful & $4(4.9)$ & $3(5.5)$ & $3(1.8)$ & $10(3.3)$ & \\
\hline \multicolumn{6}{|l|}{ Quit variables } \\
\hline \multicolumn{6}{|l|}{ Quit attempts, n (\%) } \\
\hline No & $52(64.2)$ & $44(80.0)$ & $137(83.0)$ & $233(77.4)$ & \multirow[t]{2}{*}{.004} \\
\hline Yes & $29(35.8)$ & $11(20.0)$ & $28(17.0)$ & $68(22.6)$ & \\
\hline \multicolumn{6}{|l|}{ Quit intentions, n (\%) } \\
\hline I'll quit JUUL within a year & $17(21.0)$ & $10(18.2)$ & $41(24.8)$ & $68(22.6)$ & \multirow[t]{2}{*}{.55} \\
\hline I will continue to use/don't know & $64(79.0)$ & $45(81.8)$ & $124(75.2)$ & $233(77.4)$ & \\
\hline Quit importance, mean (SD) ${ }^{\mathrm{d}}$ & $4.1(3.0)$ & $4.1(2.8)$ & $4.2(2.6)$ & $4.1(2.8)$ & .98 \\
\hline Quit confidence, mean (SD) $^{d}$ & $6.4(2.7)$ & $5.6(2.8)$ & $5.6(2.7)$ & $5.8(2.7)$ & .07 \\
\hline
\end{tabular}

Abbreviation: PSECDI, Penn State Electronic Cigarette Dependence Index.

${ }^{a}$ Data source: A 30-minute survey was developed by the study team and administered at 3 time points: July 2019, January 2020 , and April 2020.

${ }^{b} P$ values determined from variable comparisons across smoking status using 1-way analyses of variance and $x^{2}$ tests.

${ }^{c}$ Scores range from 0 to 20 , where higher scores indicate greater dependence. Levels of dependence were categorized as not dependent (0-3), low dependence (4-8), medium dependence (9-12), and high dependence ( $\geq 13)(17)$.

d A 10-point Likert scale ranging from "not at all" to "very." Scores categorized into low (1-3), medium (4-7), and high (8-10) to simplify analysis. 
Table 2. Results of Logistic Regression Models of JUUL Addictiveness Perception Among US Adult JUUL Users ( $\mathrm{N}=301)$, 2019-2020

\begin{tabular}{|c|c|c|c|}
\hline \multirow[b]{2}{*}{ Variable } & \multirow[b]{2}{*}{$\mathrm{X}^{2} P$ value } & \multicolumn{2}{|l|}{ Odds ratio $(95 \% \mathrm{Cl})$} \\
\hline & & Moderately vs not at all & Very vs not at all \\
\hline Age & .001 & $1.05(0.10-1.12)$ & $0.98(0.09-1.05)$ \\
\hline \multicolumn{4}{|l|}{ Sex } \\
\hline Male & \multirow[t]{2}{*}{.004} & $0.95(0.03-1.12)$ & $0.34(0.01-1.06)$ \\
\hline Female & & 1 [Reference] & 1 [Reference] \\
\hline \multicolumn{4}{|l|}{ Smoking status } \\
\hline Current & \multirow[t]{3}{*}{.002} & $1.42(0.04-5.49)$ & $4.02(0.08-20.19)$ \\
\hline Former & & $4.13(1.43-11.94)^{b}$ & $11.66(3.25-41.88)^{\mathrm{c}}$ \\
\hline Never & & 1 [Reference] & 1 [Reference] \\
\hline \multicolumn{4}{|c|}{ PSECDI dependence level $^{d}$} \\
\hline Low & \multirow[t]{4}{*}{$<.001$} & $1.69(0.06-4.60)$ & $5.46(0.94-31.55)$ \\
\hline Medium & & $6.31(1.57-25.39)^{b}$ & $38.33(5.33-275.41)^{\mathrm{c}}$ \\
\hline High & & $2,356,778(1,077,123-5,156,700)^{\mathrm{c}, \mathrm{e}}$ & $44,203,672(2,020,259-9,671,854)^{c, e}$ \\
\hline Not dependent & & 1 [Reference] & 1 [Reference] \\
\hline
\end{tabular}

Abbreviation: PSECDI, Penn State Electronic Cigarette Dependence Index.

${ }^{a}$ A 30-minute survey was developed by the study team and administered at 3 time points: July 2019, January 2020, and April 2020. The question on addictiveness was, "Overall, how addictive do you believe using a JUUL is?" with response options "not at all addictive," "moderately addictive," and "very addictive" Estimates of each variable level were determined by using binomial and multinomial logistic regression models. Akaike information criterion $=432.9$; residual deviance $=400.9$.

b $P<.01$; determined by $x^{2}$ test.

${ }^{\mathrm{c}} P<.001$; determined by $x^{2}$ test.

${ }^{d}$ Scores range from 0 to 20 , where higher scores indicate greater dependence. Levels of dependence were categorized as not dependent (0-3), low dependence (4-8), medium dependence (9-12), and high dependence $(\geq 13)(17)$.

${ }^{\mathrm{e}}$ The odds ratios for high dependence are large because we modeled the log-odds as a linear function of each variable and, therefore, applied an exponential function to the odds ratio calculations. These estimates are reliable and not biased by outliers.

The opinions expressed by authors contributing to this journal do not necessarily reflect the opinions of the U.S. Department of Health and Human Services, the Public Health Service, the Centers for Disease Control and Prevention, or the authors' affiliated institutions. 
Table 3. Results of Logistic Regression Models of Perceived Harm of JUUL, Compared With Smoking, Among US Adult JUUL Users (N = 301), 2019-2020

\begin{tabular}{|c|c|c|c|c|}
\hline \multirow[b]{2}{*}{ Variable } & \multirow[b]{2}{*}{$x^{2} P$ value } & \multicolumn{3}{|l|}{ Odds ratio $(95 \% \mathrm{Cl})$} \\
\hline & & Less vs much less harmful & Just as much vs much less harmful & Much more vs much less harmful \\
\hline \multicolumn{5}{|l|}{ Sex } \\
\hline Male & \multirow[t]{2}{*}{.01} & $1.20(0.66-2.17)$ & $0.72(0.35-1.47)$ & $0.26(0.06-1.09)$ \\
\hline Female & & 1 [Reference] & 1 [Reference] & 1 [Reference] \\
\hline \multicolumn{5}{|l|}{ Smoking status } \\
\hline Current & \multirow[t]{3}{*}{.02} & $4.56(1.75-11.88)^{b}$ & $3.81(1.26-11.52)^{\mathrm{C}}$ & $3.78(0.64-22.45)$ \\
\hline Former & & $2.55(1.32-4.94)^{b}$ & $1.71(0.77-3.78)$ & $0.65(0.12-3.46)$ \\
\hline Never & & 1 [Reference] & 1 [Reference] & 1 [Reference] \\
\hline Days of JUUL use in past 30 days & .09 & $0.98(0.94-1.01)$ & $0.96(0.92-1.00)$ & $0.92(0.85-0.99)^{c}$ \\
\hline $\begin{array}{l}\text { Months of e-cigarette use, square } \\
\text { root }\end{array}$ & .046 & $0.90(0.79-1.02)$ & $0.83(0.70-0.97)^{c}$ & $1.12(0.83-1.51)$ \\
\hline
\end{tabular}

${ }^{a}$ A 30-minute survey was developed by the study team and administered at 3 time points: July 2019, January 2020, and April 2020. The question on harm perception was, "Compared to smoking, would you say that JUUL use is ... " with response options "much less harmful," "less harmful," "just as harmful," or "much more harmful." Estimates of each variable level were determined by using binomial and multinomial logistic regression models. Akaike information criterion = 688.6; residual deviance $=652.6$.

${ }^{\mathrm{b}} P<.01$; determined by $x^{2}$ test.

${ }^{c} P<.001$; determined by $x^{2}$ test. 
Table 4. Results of Logistic Regression Models of Quit Intentions Among US Adult JUUL Users (N = 301), 2019-2020

\begin{tabular}{|c|c|c|}
\hline Variable & $X^{2} P$ value & I'll quit vs l'll continue \\
\hline \multicolumn{3}{|c|}{ Perceived addictiveness of JUUL } \\
\hline Moderately addictive & \multirow[t]{3}{*}{$<.001$} & $3.36(0.88-22.35)$ \\
\hline Very addictive & & $12.46(2.92-88.24)^{b}$ \\
\hline Not at all & & 1 [Reference] \\
\hline \multicolumn{3}{|c|}{ Perceived harm of JUUL, compared with smoking } \\
\hline Less harmful & \multirow[t]{4}{*}{.14} & $0.68(0.33-1.47)$ \\
\hline Just as much harm & & $0.86(0.36-2.03)$ \\
\hline Much more harm & & $3.37(0.77-15.91)$ \\
\hline Much less harm & & 1 [Reference] \\
\hline JUUL use times per day, log & .03 & $0.70(0.49-0.97)^{\mathrm{C}}$ \\
\hline Months of JUUL use & .51 & $1.08(0.85-1.38)$ \\
\hline Months of e-cigarette use & .05 & $0.85(0.72-1.00)$ \\
\hline \multicolumn{3}{|l|}{ Survey time point } \\
\hline Time point 2 & \multirow[t]{3}{*}{.06} & $2.26(1.01-5.48)$ \\
\hline Time point 3 & & $2.75(1.16-7.00)^{\mathrm{C}}$ \\
\hline Time point 1 & & 1 [Reference] \\
\hline
\end{tabular}

${ }^{\text {a }}$ A 30-minute survey was developed by the study team and administered at 3 time points: July 2019, January 2020, and April 2020. The question on quit intentions was, "Are you planning to continue using your JUUL electronic cigarette for at least the next year, or quit within that time frame?" with response options "I'll quit using JUUL within a year," "I plan to continue using the JUUL," or "Don't know." Estimates of each variable level were determined by using binomial and multinomial logistic regression models. Akaike information criterion $=306.5$; residual deviance $=284.5$.

${ }^{\mathrm{b}} P<.01$.

${ }^{\mathrm{c}} P<.05$. 
Table 5. Results of Logistic Regression Models of Quit Attempts Among US Adult JUUL Users ( $\mathrm{N}=301), 2019-2020^{a}$

\begin{tabular}{|c|c|c|}
\hline Variable & $\mathrm{X}^{2} P$ value & Yes vs no \\
\hline \multicolumn{3}{|l|}{ Perceived addictiveness of JUUL } \\
\hline Moderately addictive & \multirow[t]{3}{*}{.03} & $5.64(0.99-107.16)$ \\
\hline Very addictive & & $10.56(1.62-212.12)^{b}$ \\
\hline Not at all & & 1 [Reference] \\
\hline \multicolumn{3}{|c|}{ Perceived harm of JUUL, compared with smoking } \\
\hline Less harmful & \multirow[t]{4}{*}{.10} & $0.68(0.30-1.52)$ \\
\hline Just as much harm & & $1.83(0.76-4.49)$ \\
\hline Much more harm & & $0.97(0.17-5.26)$ \\
\hline Much less harm & & 1 [Reference] \\
\hline \multicolumn{3}{|l|}{ Smoking status } \\
\hline Current & \multirow[t]{3}{*}{.007} & $0.35(0.14-0.87)^{b}$ \\
\hline Former & & $0.32(0.15-0.66)^{c}$ \\
\hline Never & & 1 [Reference] \\
\hline \multicolumn{3}{|l|}{ PSECDI dependence level ${ }^{d}$} \\
\hline Low & \multirow[t]{4}{*}{$<.001$} & $2.96(0.81-14.52)$ \\
\hline Medium & & $15.16(4.26-75.04)^{\mathrm{e}}$ \\
\hline High & & $8.85(2.18-47.39)^{\mathrm{C}}$ \\
\hline Not dependent & & 1 [Reference] \\
\hline Days of JUUL use in past 30 days & .002 & $0.94(0.90-0.98)^{c}$ \\
\hline
\end{tabular}

Abbreviation: PSECDI, Penn State Electronic Cigarette Dependence Index.

${ }^{a}$ A 30-minute survey was developed by the study team and administered at 3 time points: July 2019, January 2020, and April 2020. The question on quit attempt was, "Have you ever tried to quit using your JUUL electronic cigarette?" with response options no or yes. Estimates of each variable level were determined by using binomial and multinomial logistic regression models. Akaike information criterion $=280.9$; residual deviance $=256.9$.

${ }^{\mathrm{b}} P<.05$

${ }^{c} P<.01$.

${ }^{\mathrm{d}}$ Scores range from 0 to 20 , where higher scores indicate greater dependence. Levels of dependence were categorized as not dependent (0-3), low dependence $(4-8)$, medium dependence (9-12), and high dependence ( $\geq 13)(17)$.

${ }^{\mathrm{e}} P<.001$. 
Table 6. Results of Logistic Regression Models of Quit Importance and Quit Confidence Among US Adult JUUL Users ( $\mathrm{N}=301)$, 2019-2020

\begin{tabular}{|c|c|c|c|}
\hline Variable & $\mathrm{X}^{2} P$ value & Moderate vs low & High vs low \\
\hline \multicolumn{4}{|l|}{ Quit importance $^{\text {b }}$} \\
\hline \multicolumn{4}{|l|}{ Survey time point } \\
\hline Time point 2 & \multirow[t]{3}{*}{.04} & $2.21(1.07-4.56)^{c}$ & $1.16(0.41-3.33)$ \\
\hline Time point 3 & & $3.40(1.56-7.42)^{d}$ & $1.29(0.42-3.99)$ \\
\hline Time point 1 & & 1 [Reference] & 1 [Reference] \\
\hline Age & .09 & $0.99(0.96-1.02)$ & $1.04(0.99-1.09)$ \\
\hline \multicolumn{4}{|l|}{ Place of JUUL purchase } \\
\hline Vape shop & \multirow[t]{5}{*}{.03} & $1.03(0.50-2.10)$ & $1.20(0.42-3.38)$ \\
\hline Tobacco store & & $0.75(0.28-2.02)$ & $0.62(0.15-2.48)$ \\
\hline Online & & $0.54(0.25-1.18)$ & $0.19(0.42-0.81)^{c}$ \\
\hline Did not purchase & & $0.33(0.05-2.01)$ & $3.97(0.70-22.45)$ \\
\hline Gas station & & 1 [Reference] & 1 [Reference] \\
\hline \multicolumn{4}{|l|}{ Perceived addictiveness of JUUL } \\
\hline Moderately addictive & \multirow[t]{3}{*}{.02} & $1.80(0.58-5.64)$ & $1.95(0.18-21.27)$ \\
\hline Very addictive & & $1.33(0.35-5.04)$ & $7.05(0.54-92.91)$ \\
\hline Not at all addictive & & 1 [Reference] & 1 [Reference] \\
\hline \multicolumn{4}{|c|}{ Perceived harm of JUUL, compared with smoking } \\
\hline Less harmful & \multirow[t]{4}{*}{$<.001$} & $4.08(1.96-8.47)^{\mathrm{e}}$ & $1.65(0.53-5.16)$ \\
\hline Just as much harm & & $4.30(1.79-10.35)^{d}$ & $5.84(1.71-19.92)^{d}$ \\
\hline Much more harm & & $11.21(0.95-131.79)$ & $28.20(2.46-323.29)^{d}$ \\
\hline Much less harm & & 1 [Reference] & 1 [Reference] \\
\hline \multicolumn{4}{|l|}{ PSECDI dependence level ${ }^{\dagger}$} \\
\hline Low & \multirow[t]{4}{*}{.002} & $3.70(1.41-9.72)^{d}$ & $3.10(0.59-16.28)$ \\
\hline Medium & & $4.96(1.78-13.81)^{d}$ & $13.75(2.48-76.26)^{d}$ \\
\hline High & & $4.47(1.36-14.66)^{c}$ & $10.43(1.66-65.42)^{\mathrm{C}}$ \\
\hline Not dependent & & 1 [Reference] & 1 [Reference] \\
\hline Days of JUUL use in past 30 days & .001 & $0.96(0.92-0.99)^{c}$ & $0.90(0.85-0.96)^{e}$ \\
\hline Months of e-cigarette use & .006 & $0.81(0.71-0.93)^{d}$ & $0.84(0.70-1.00)$ \\
\hline
\end{tabular}

Abbreviation: PSECDI, Penn State Electronic Cigarette Dependence Index.

${ }^{a}$ A 30-minute survey was developed by the study team and administered at 3 time points: July 2019, January 2020, and April 2020. Estimates of each variable level were determined by using binomial and multinomial logistic regression models.

${ }^{\mathrm{b}}$ The question on quit importance was, "How important is it for you to quit electronic cigarette use now?" Akaike information criterion = 552.5; residual deviance = 480.5 .

${ }^{c} P<.05$; determined by $x^{2}$ test.

${ }^{d} P<.01$; determined by $X^{2}$ test.

${ }^{\mathrm{e}} P<.001$; determined by $x^{2}$ test.

${ }^{f}$ Scores range from 0 to 20 , where higher scores indicate greater dependence. Levels of dependence were categorized as not dependent (0-3), low dependence (4-8), medium dependence (9-12), and high dependence ( $\geq 13)(17)$.

${ }^{\mathrm{g}}$ The question on quit confidence was, “How confident are you that you could quit electronic cigarette use now?" Akaike information criterion = 605.5; residual deviance $=589.5$. 
(continued)

Table 6. Results of Logistic Regression Models of Quit Importance and Quit Confidence Among US Adult JUUL Users ( $\mathrm{N}=301)$, 2019-2020

\begin{tabular}{|c|c|c|c|}
\hline Variable & $X^{2} P$ value & Moderate vs low & High vs low \\
\hline \multicolumn{4}{|l|}{ Quit confidence $^{\mathrm{g}}$} \\
\hline \multicolumn{4}{|c|}{ PSECDI dependence level ${ }^{f}$} \\
\hline Low & \multirow[t]{4}{*}{$<.001$} & $2.00(0.63-6.33)$ & $0.68(0.23-2.02)$ \\
\hline Medium & & $0.98(0.33-2.90)$ & $0.14(0.04-0.41)^{\mathrm{e}}$ \\
\hline High & & $0.50(0.16-1.56)^{c}$ & $0.08(0.02-0.26)^{\mathrm{e}}$ \\
\hline Not dependent & & 1 [Reference] & 1 [Reference] \\
\hline
\end{tabular}

Abbreviation: PSECDI, Penn State Electronic Cigarette Dependence Index.

${ }^{a}$ A 30-minute survey was developed by the study team and administered at 3 time points: July 2019, January 2020, and April 2020. Estimates of each variable level were determined by using binomial and multinomial logistic regression models.

${ }^{\mathrm{b}}$ The question on quit importance was, "How important is it for you to quit electronic cigarette use now?" Akaike information criterion $=552.5$; residual deviance = 480.5 .

${ }^{c} P<.05$; determined by $x^{2}$ test.

${ }^{\mathrm{d}} P<.01$; determined by $X^{2}$ test.

e $P<.001$; determined by $x^{2}$ test.

${ }^{f}$ Scores range from 0 to 20 , where higher scores indicate greater dependence. Levels of dependence were categorized as not dependent (0-3), low dependence (4-8), medium dependence (9-12), and high dependence ( $\geq 13)(17)$.

${ }^{g}$ The question on quit confidence was, "How confident are you that you could quit electronic cigarette use now?" Akaike information criterion = 605.5; residual deviance $=589.5$. 